\title{
Epidemiology of Hospitalized Patients with Babesiosis, United States, 2010-2016
}

\author{
Evan M. Bloch, Jonathan R. Day, Peter J. Krause, \\ Anne Kjemtrup, Sheila F. O’Brien, Aaron A.R. Tobian, Ruchika Goel
}

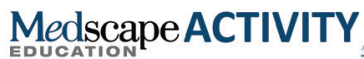

In support of improving patient care, this activity has been planned and implemented by Medscape, LLC and Emerging Infectious Diseases. Medscape, LLC is jointly accredited by the Accreditation Council for Continuing Medical Education (ACCME), the Accreditation Council for Pharmacy Education (ACPE), and the American Nurses Credentialing Center (ANCC), to provide continuing education for the healthcare team.

Medscape, LLC designates this Journal-based CME activity for a maximum of 1.00 AMA PRA Category 1 Credit(s) ${ }^{\mathrm{TM}}$. Physicians should claim only the credit commensurate with the extent of their participation in the activity.

Successful completion of this CME activity, which includes participation in the evaluation component, enables the participant to earn up to $1.0 \mathrm{MOC}$ points in the American Board of Internal Medicine's (ABIM) Maintenance of Certification (MOC) program. Participants will earn MOC points equivalent to the amount of CME credits claimed for the activity. It is the CME activity provider's responsibility to submit participant completion information to ACCME for the purpose of granting ABIM MOC credit.

All other clinicians completing this activity will be issued a certificate of participation. To participate in this journal CME activity: (1) review the learning objectives and author disclosures; (2) study the education content; (3) take the post-test with a $75 \%$ minimum passing score and complete the evaluation at http://www.medscape.org/journal/eid; and (4) view/print certificate. For CME questions, see page XXX.

Release date: January 20, 2022; Expiration date: January 20, 2023

\section{Learning Objectives}

Upon completion of this activity, participants will be able to:

- Assess the pathology and outcomes of babesiosis

- Distinguish the characteristics of patients with babesiosis

- Analyze the most common season and geographic areas for babesiosis in the US

- Identify the most common complication of babesiosis in the current study

\section{CME Editor}

Karen L. Foster, Technical Writer/Editor, Emerging Infectious Diseases. Disclosure: Karen L. Foster has disclosed no relevant financial relationships.

\section{CME Author}

Charles P. Vega, MD, Health Sciences Clinical Professor of Family Medicine, University of California, Irvine School of Medicine, Irvine, California. Disclosure: Charles $P$. Vega, MD, has disclosed the following relevant financial relationships: served as an advisor or consultant for Johnson \& Johnson.

\section{Authors}

Evan M. Bloch, MBChB; Jonathan R. Day, MD, PharmD; Peter J. Krause, MD; Anne Kjemtrup, DVM, MPVM, PhD; Sheila F. O'Brien, PhD; Aaron A.R. Tobian, MD, PhD; and Ruchika Goel, MD, MPH.

Author affiliations: Johns Hopkins School of Medicine, Baltimore, Maryland, USA (E.M. Bloch, A.A.R. Tobian, R. Goel); University of lowa Health Care, lowa City, lowa, USA (J.R. Day); Simmons Cancer Institute at Southern Illinois University, Springfield, Illinois, USA (J.R. Day, R. Goel); Yale School of Public Health and Yale School of Medicine, New Haven, Connecticut USA (P.J. Krause); California Department of Public Health, Sacramento, California, USA (A. Kjemtrup); Canadian Blood Services, Ottawa, Ontario, Canada (S.F. O’Brien) 
Babesia spp. are tickborne parasites that cause the clinical infection babesiosis, which has an increasing incidence in the United States. We performed an analysis of hospitalizations in the United States during 2010-2016 in which babesiosis was listed as a diagnosis. We used the National Inpatient Sample database to characterize the epidemiology of Babesia-associated admissions, reflecting severe Babesia-related disease. Over a 7-year period, a total of 7,818 hospitalizations listed babesiosis as a primary or secondary admitting diagnosis. Hospitalizations were seasonal $(71.2 \%$ occurred during June-August) and situated overwhelmingly in the Northeast and Midwest. The patients were predominantly male and of advanced age, which is consistent with the expected epidemiology. Despite a higher severity of illness in more than (58.5\%), the mortality rate was low $(1.6 \%)$. Comparison with state reporting data suggests that the number of hospitalized persons with babesiosis increased modestly during the observation period.

$B$ abesia spp. are tickborne intraerythrocytic apicomplexan parasites responsible for the clinical infection babesiosis. Babesia microti, the leading cause of human babesiosis, is endemic in the northeastern and north-midwestern United States (1). Although infection in immunocompetent adults may be mild or even subclinical, manifesting as a self-limiting virallike illness (i.e., fever, headache, myalgia, fatigue), risk for severe disease and complications exists in certain patient populations (i.e., the very young, the elderly, persons with asplenia, and others with immunosuppression). Like Plasmodium parasites that cause malaria, Babesia spp. infect erythrocytes and induce hemolysis. Clinical complications include severe anemia, renal failure, cardiorespiratory failure, and death (1). Babesia spp. also are readily transmissible by transfusion of infected erythrocytes. Given that anemia is the major indication for erythrocyte transfusion, coupled with the high proportion of patients at high risk for severe disease in the transfused population, transfusion-transmitted babesiosis has a death rate of $\approx 20 \%(1,2)$.

Reported cases of babesiosis and other tickborne diseases are increasing (3-5). Postulated reasons for the increase include expansion of the geographic range of tick vector population, increase in deer (and consequent tick) populations, encroachment of humans into Babesia zoonotic habitats, climate change, and other ecologic changes that contribute to a rise in incidence of Babesia infection $(6,7)$. Babesiosis was designated a nationally notifiable disease in the United States in 2011, meaning that states where it was reportable were charged to voluntarily notify the Centers for Disease Control and Prevention (CDC) of cases. As of 2015, babesiosis was reportable in 33 states $(8,9)$. Although an increase in babesiosis cases has been reported, whether the increase includes primarily outpatients, hospitalized case-patients, or both is uncertain. To test whether hospitalized babesiosis patients are increasing, we analyzed hospitalizations in the United States in which babesiosis was listed as a diagnosis, using the National (Nationwide) Inpatient Sample (NIS) database, which offers a representative sampling of US-based hospitals. This analysis enabled characterization of the epidemiology of admissions, reflecting severe Babesia-related disease.

\section{Methods}

This study uses 7 years of data (i.e., 2010-2016) from the NIS, the largest publicly available inpatient healthcare database in the United States. The NIS was developed as a federal-state-industry partnership by the Agency for Healthcare Research and Quality for the Healthcare Cost and Utilization Project (HCUP). Data before 2012 used a 20\% stratified probability sample of hospitals rather than discharges (10). After a redesign in 2012, the NIS adopted a sampling design that uses a stratified probability sample of $20 \%$ of all HCUP participating hospital discharges for each calendar year. This sampling scheme is estimated to cover $90 \%-97 \%$ of the US population across the different years (11). The unit of analysis is a single hospitalization and not a specific patient; therefore, a single patient may be represented in multiple observations. Observations are self-weighted and calculated by strata, which are defined by census division (categorized as census region before 2012), bed size, location, teaching status, and hospital ownership.

The NIS provides de-identified discharge data without individual patient or hospital-level identifiers. These data include 1 primary or principal diagnosis code, up to 29 secondary diagnosis codes, and up to 15 procedure codes. The principal diagnosis is the primary reason for admission and is coded in the first diagnosis field. The number of diagnoses and associated data elements was increased from 15 to 25 in 2009 and from 25 to 30 beginning in 2014. We have captured this change in our analysis.

Demographic details extracted from the database were age, sex, and race. Hospital-level characteristics were location (urban vs rural), academic designation (teaching vs. nonteaching), and bed size. Hospitals were categorized as small, medium, or large according to the criteria defined by HCUP, which were based on region, urban-rural designation, and teaching status (12). Other variables included admission and discharge status, total charges, expected payment 
source, length of hospital stay, and hospital characteristics. The NIS database uses All Patient Refined Diagnosis Related Groups (APR-DRGs), a validated inpatient classification system that is widely used in the United States to assess severity of illness and risk for death during hospitalization using multiple variables. The risk for death and severity of illness are ranked on a scale of 1 to 4 , corresponding to mild, moderate, major, and extreme.

We used diagnosis and procedure codes from the International Classification of Diseases, Ninth Revision, Clinical Modification (ICD-9-CM), from 2010 through the third quarter of 2015. After the third quarter of 2015, billing codes switched from ICD-9$\mathrm{CM}$ to the International Classification of Diseases, Tenth Revision, Clinical Modification (ICD-10-CM). Babesiosis cases were identified by ICD-9 code 088.82 or ICD-10 code B60.0. Transfusion-transmitted babesiosis infections could not be identified independently because there are no specific ICD-9 or ICD-10 codes for that diagnosis. We described demographic and clinical characteristics as counts, percentages, mean (SD) and median (interquartile range) as appropriate. We stratified results into ICD-9 (2010-2015q3) and ICD-10 (2015q4-2016) data. We analyzed the geographic distribution, demographics. and seasonality of Babesia-related hospitalizations and stratified and analyzed hospitalizations by the leading regional divisions. We calculated transfusions and incidence of erythrocyte exchanges during admissions as the binomial proportion of encounters during which $\geq 1$ blood product was issued or erythrocyte exchange was performed. We performed logistic regression to compare the incidence of various clinical co-morbidities and outcomes. All $\mathrm{p}$ values were 2-tailed and statistical significance was set at $\mathrm{p}<0.05$. We analyzed data using Stata version 15 (StataCorp LLC, https://www. stata.com), using survey analysis commands applying the sampling weights as determined by HCUP.

We also performed a graphical comparison of the number and incidence of babesiosis cases reported to CDC during 2011-2016 to compare the overall trends in reporting. The CDC data that were included were reported by individual state health departments; cases were reported by the state of residence, which might not have been the state of exposure.

To test specificity, we performed a sensitivity analysis that restricted the assessment to hospitalizations in which babesiosis was listed in the top 5 diagnoses. The analysis also excluded admissions associated with a primary diagnosis of Lyme disease.

Given that the NIS is a de-identified, publicly available dataset, this study was deemed exempt from review from the Johns Hopkins Institutional Review Board. This analysis was conducted in accordance with the HCUP data use agreement guidelines.

\section{Results}

During a 7-year period, babesiosis was listed as an admitting diagnosis for 7,818 hospitalizations, of which $4,648(59.5 \%)$ listed babesiosis as a primary diagnosis and $3,170(40.5 \%)$ as a secondary diagnosis (Table 1, https://wwwnc.cdc.gov/EID/article/28/2/210213-T1.htm). Annual hospitalizations varied by year, from 676 in 2010 to 1415 in 2013 (Figure). For all hospitalizations, the median age of patients was 67 years (interquartile range 55-77 years); 5,001 (64\%) of the associated patients were male, and 6,024 (80.1\%) were White.

Of all hospitalizations, 2,325 (71.2\%) occurred in summer (June-August); 6,616 (92.7\%) occurred in the Northeast and Midwest. New England (1,150 [44.1\%] hospitalizations) and Mid-Atlantic (1,115 [42.7\%] hospitalizations) were the leading regions. The admitting hospitals were predominantly urban $(6,529[91.4 \%])$, and admissions were overwhelmingly nonelective $(7,452$ [95.4\%]).

A greater severity of illness was reported in 4,574 (58.5\%) hospitalizations; risk for death was assessed as major or extreme for 1,339 (17.2\%) hospitalizations (Table 2). The leading complications included acute renal failure (1,594 [20.4\%] hospitalizations), respiratory failure (528 [6.8\%]), and cardiac failure (270 [3.5\%]) (Table 3). A total of 128 deaths occurred over the 7-year period, representing $1.6 \%$ of all babesiosis-associated admissions. Babesiosis was the primary hospital-associated diagnosis for 20 of those deaths and secondary for the other 108 deaths. Similar to the distribution of all hospitalizations, most deaths occurred in the Northeast (89 [69.5\%]) and the Midwest (10 [7.8\%]).

Babesiosis-related deaths were significantly associated with acute renal failure $(p<0.001)$, acute respiratory failure $(p<0.001)$, and disseminated intravascular coagulation $(p=0.001)$ when compared with nonfatal hospitalizations. The mean length of stay was $5.8 \pm 7.3$ days (Table 3 ). The aggregate national bill for the 7-year period for a principal diagnosis of babesiosis was $>\$ 170$ million USD $(\$ 171,281,170)$, averaging \$24.4 million USD per year, and the mean hospital charge for a Babesia-associated admission was $\$ 36,850$.

At least 1 erythrocyte transfusion was reported in 1,560 (20\%) hospital admissions (Table 3). Transfusion of other blood products was comparatively rare. Hospitalizations in which erythrocyte transfusions were 


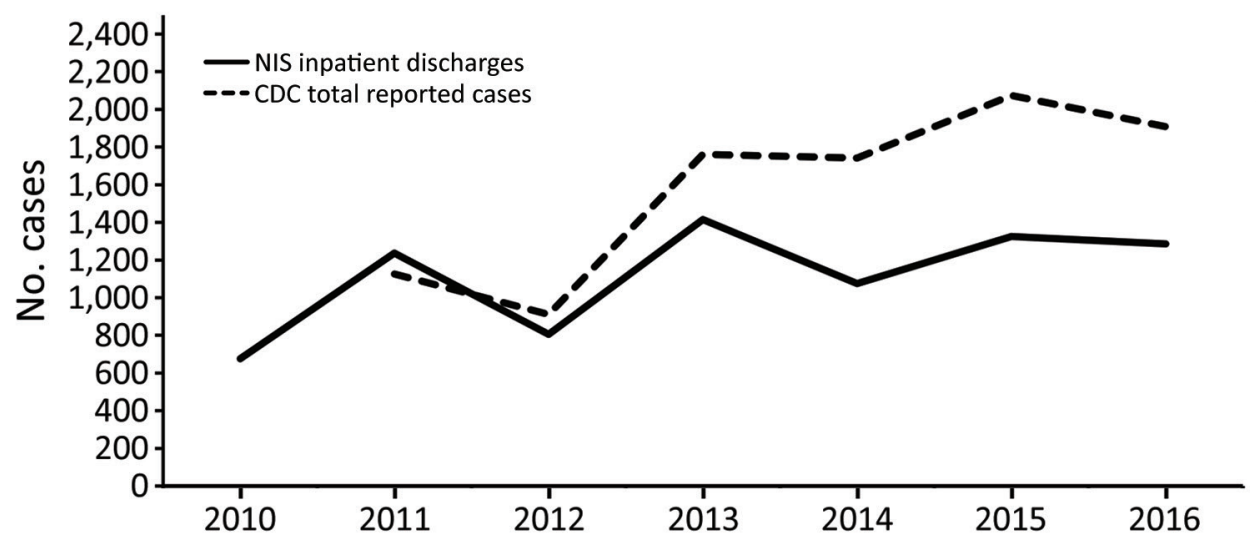

Figure. Cases of babesiosis in the United States, 2010-2016, CDC versus NIS data. CDC, Centers for Disease Control and Prevention; NIS, National Inpatient Sample.

reported were associated with severe illness. Major or extreme severity of illness was reported in $\approx 80 \%$ in the erythrocyte transfusion group, compared with $53 \%$ in those for which erythrocyte transfusions were not reported. Furthermore, $59.9 \%$ of the cases in the erythrocyte transfusion group were assessed as having a major or extreme risk for death in comparison with $32.3 \%$ for those in which no erythrocyte transfusion was reported. A significantly higher rate of death was observed in the transfusion group $(3.18 \%$ vs. $1.27 \% ; p=0.02$ ). Erythrocyte exchange (i.e., erythrocytapheresis) was performed in $80(1 \%)$ admissions. Most admissions in which erythrocytapheresis was undertaken were associated with high illness severity. Specifically, $18.3 \%$ were associated with moderate, $18.8 \%$ with major, and $63 \%$ with extreme severity of illness.

A total of 1,953 (25\%) babesiosis-related hospitalizations listed Lyme disease as a concurrent diagnosis; 276 (3.5\%) listed Lyme disease as a primary diagnosis. Neither disease severity nor mortality differed for those hospitalizations in which only babesiosis was listed, compared with those hospitalizations in which both babesiosis and Lyme disease were listed $(p>0.05)$. Rates of respiratory failure, heart failure, disseminated intravascular coagulation, and mean length of stay did not differ between those with and without a concurrent diagnosis of Lyme disease.

Other notable concurrent diagnoses in babesiosis-associated hospitalizations were anaplasmosis and ehrlichiosis (658 [8.4\%]); these 2 entities are combined because it was not possible to distinguish them on the basis of the coding in use. Malaria, a clinical and morphologic diagnostic mimic of babesiosis, was reported in $52(0.7 \%)$ babesiosis-associated hospitalizations. In 560 (7.2\%) of babesiosis-associated hospitalizations, the patients were noted to have decreased splenic function or were asplenic.
When we restricted the hospitalizations to those in which babesiosis was listed in the top 5 diagnoses and a primary diagnosis of Lyme disease was excluded, the number of admissions changed from 7,818 to 6,903 . However, all analyses remained comparable (Appendix Tables 1-3, https://wwwnc.cdc.gov/ EID/article/28/2/21-0213-App1.pdf).

\section{Discussion}

Our findings offer a nationally representative estimate of in-hospital babesiosis in the United States. During a 7-year period, most $(\approx 85 \%)$ Babesia-related hospitalizations occurred in the New England and Mid-Atlantic states, and two thirds occurred in the summer (June-August). More than half of all patients were $\geq 65$ years of age, and almost two thirds were male. A concurrent diagnosis of Lyme disease was reported in one quarter of all babesiosis-related hospitalizations. Reported clinical complications, notably acute renal failure, were common. Consistent with a selection for severe cases that warrant hospital admission, a high proportion of patients experienced major or extreme severity of illness and were deemed to be at high risk for death. Nonetheless, the overall mortality rate was low. Despite an upward trend in annual cases of babesiosis that have been reported to CDC, Babesia-related hospitalizations appeared stable or modestly increasing during the study period.

The data on geographic distribution, demographics, and seasonality of Babesia-related hospitalizations in this report are consistent with what is known about the parasite's general epidemiology in the United States. Specifically, B. microti is widely endemic in the Northeast and upper Midwest. B. microti is the most common species causing human babesiosis in the United States and worldwide. Other species (e.g., $B$. duncani) and variants (e.g., $B$. divergens-like/MO-1) have been reported in the United States but are comparatively rare (13-16). Male predominance may be 
Hospitalized Patients with Babesiosis

Table 3. Clinical outcomes and healthcare use in patients with babesiosis-associated hospitalizations, United States, 2010-2016*

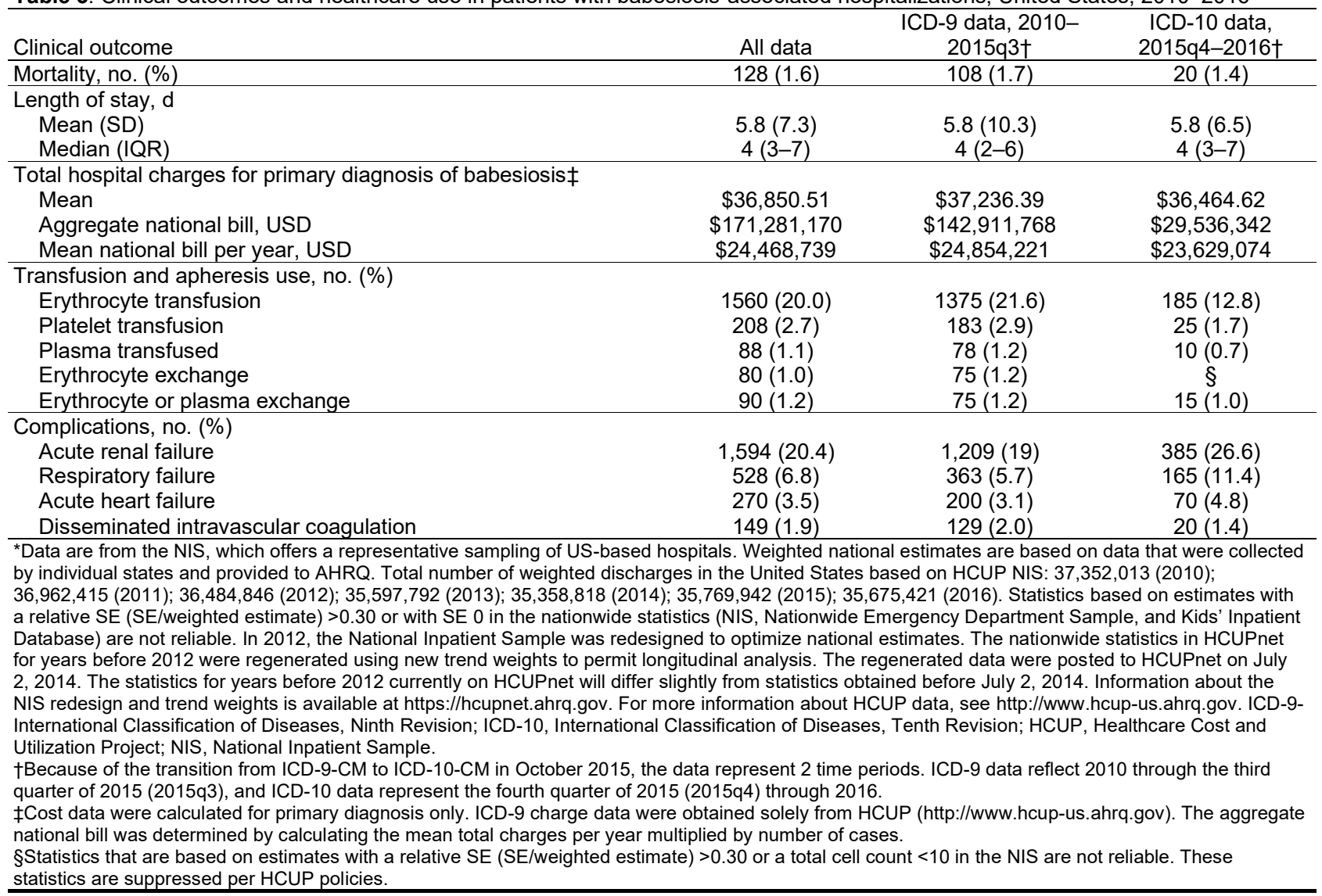

A stable or modest increase in severe babesiosis (i.e., hospitalizations) does not correlate well with the general epidemiology of this infection, whereby an increase in cases of babesiosis has been observed over the past 3 decades (22). Several factors may contribute to a general increase, such as increased geographic spread of the disease, allied with increased recognition of the disease. A group of investigators have used tick surveillance to estimate the geographic range and disease burden of babesiosis (23-26). A close association between B. microti in ticks and reported rates of human infection was demonstrated in babesiosis-endemic areas but not in areas of emerging disease, suggesting underreporting outside of established areas of endemicity (25). Furthermore, that the overall reported cases (which included the hospitalized patients) were only $\approx 50 \%$ more may suggest that cases are either not reported or not recognized.

We found that clinical complications of babesiosis in our study population were common, but the overall mortality rate $(\approx 1.3 \%)$ was low. The observed rates of clinical complications in our study differ from prior reports, which tended to cite pulmonary sequelae (e.g., pulmonary edema, acute respiratory failure, and acute respiratory distress syndrome) as the most common severe complication of babesiosis (prevalence ranging from $6.3 \%$ to $43 \%)(17,27-29)$. By contrast, acute renal failure was the leading complication in our population (prevalence of $20.4 \%$ for acute renal failure, compared with $6.8 \%$ for acute respiratory failure). Previously published prevalence estimates for renal failure have ranged from $4.3 \%$ to $7 \%$, the latter in immunocompromised patients $(17,27,28)$. These discrepancies could be due to differences in the definition of organ failure and the scope of prior studies. Many of the reports of organ morbidity are based on small numbers of study participants.

Similarly, previously reported death rates for babesiosis in the United States have been highly variable, from $0 \%$ to $27 \%(29,30)$. Differences by reporting source could account for the observed variability. Specifically, most studies that have reported death rates are case series, most of which have been constrained by small sample sizes. There are also differences in the populations that have been described; higher rates have been observed in asplenic patients, 
immunocompromised persons, and transfusion recipients $(2,28,30)$. Although our study offers a population-based estimate of babesiosis-related death in hospitalized patients, its findings need to be interpreted within the bounds of the acknowledged limitations pertaining to diagnostic coding and clinical imputability. Thus, data are insufficient to conclude whether death rates for babesiosis are improving; rather, the low death rate found in our study may provide a false sense of optimism regarding the disease.

Not surprisingly, erythrocyte transfusion, which was reported in one fifth of hospitalizations, was associated with a significantly higher death rate $(\approx 3 \%)$. Erythrocyte transfusions are administered for severe anemia, so they are an index of severe disease, especially for transfusion-transmitted babesiosis, which carries a high death rate $(19 \%)$ (2). Another potential risk factor for severe babesiosis is concurrent Lyme disease; previous studies have found that patients with both babesiosis and Lyme disease have more symptoms that last longer than do those with Lyme disease alone (31-33). However, the same studies failed to find a difference in the number of symptoms in patients with babesiosis and Lyme disease (i.e., co-infection), compared with patients with babesiosis alone; our data were consistent with those findings. As noted in this analysis, death was a more common outcome in admissions in which babesiosis was listed as a primary rather than as a secondary diagnosis. At least some of the secondary diagnoses are likely to be cases in which the infection was detected incidentally, where parasitemia would likely be low.

The medical care costs of babesiosis also add to the health burden of the disease. The observed charges are comparable to those associated with hospitalizations for Lyme disease; that is, the average hospital charge for a primary diagnosis of Lyme disease was $\$ 25,025.53-\$ 31,209.36$ during the study period, depending on the reporting period (ICD-9 vs. ICD-10) (data not shown). Although Lyme disease poses a greater health and economic burden, given a greater number of cases and persistence of complications of illness, deaths from Lyme disease, unlike babesiosis, are rarely encountered (34).

A limitation of our study is that the analysis is confined to hospitalized case-patients. By design, hospitalizations offer insight into the most severe cases (i.e., there is an inherent selection bias favoring severe infection). Although our findings are informative, quantifying the complete burden of disease given this highly selected sampling is difficult. Furthermore, the sampling approach does not include all hospitalized patients, nor does it include all hospitals; however, it is a validated, robust sampling approach, correlating well with other national survey methods $(35,36)$. The NIS database is not designed to capture all pediatric patients, although children who are treated in adult hospitals may be captured in these analyses. There are also technical limitations. We cannot determine whether each hospitalization was for a unique patient; a proportion may be readmissions for the same patients, although readmissions are not expected to be common. The analysis also leaves some uncertainty surrounding the relationship between babesiosis and possible concurrent diseases. For example, the observed prevalence of anaplasmosis and ehrlichiosis may reflect infection with Ehrlichia muris (also vectored by I. scapularis ticks), exposure to similar tick habitats where I. scapularis and Amblyomma ticks co-exist, or a misdiagnosis. Another limitation is the estimation of hospitalizations for transfusion-transmitted babesiosis. Unfortunately, rates of transfusion-transmitted babesiosis cannot be quantified because causality cannot be established; although one can deduce whether a transfusion was administered, it is not possible to determine whether infection was ascribed to the index transfusion using the database alone. Given the nature of this analysis, we have been cautious not to overinterpret the findings. For example, we cannot be certain whether admissions are increasing in general, whether the demographics of those who are likely to require hospitalization for babesiosis is increasing (i.e., "baby boomers"), or whether babesiosis is simply being recognized in patients because of greater awareness of symptoms.

In conclusion, we found that there has been a modest increase in hospitalized patients with babesiosis in the United States, yet the associated death rate appears to be low. Nonetheless, the overall health burden, particularly for selected patient subsets who are at risk for severe or even fatal disease, remains a concern.

E.M.B. was supported in part by National Heart Lung and Blood Institute (1K23HL151826-01). P.J.K. received support from the The Llura A. Gund Laboratory for Vector-borne Diseases and the Gordon and Llura Gund Foundation.

E.M.B. reports personal fees and nonfinancial support from Terumo BCT, Abbott Laboratories, and Grifols Diagnostics Solutions, outside of the submitted work. R.G. reports personal fees from Terumo BCT, and Alexion pharmaceuticals, outside of the submitted work. 


\section{About the Author}

Dr. Bloch is an associate professor of pathology at Johns Hopkins University School of Medicine. where he serves as associate director in the Division of Transfusion Medicine. He has a joint appointment in International Health in the Johns Hopkins Bloomberg School of Public Health. His research interests include global blood transfusion safety, particularly in the context of infectious risk and how to respond to emerging and neglected transfusion transmissible infections.

\section{References}

1. Vannier E, Krause PJ. Human babesiosis. N Engl J Med. 2012;366:2397-407. https:// doi.org/10.1056/NEJMra1202018

2. Herwaldt BL, Linden JV, Bosserman E, Young C, Olkowska D, Wilson M. Transfusion-associated babesiosis in the United States: a description of cases. Ann Intern Med. 2011;155:509-19. https://doi.org/10.7326/ 0003-4819-155-8-201110180-00362

3. Leiby DA, Gill JE. Transfusion-transmitted tick-borne infections: a cornucopia of threats. Transfus Med Rev. 2004;18:293-306. https://doi.org/10.1016/j.tmrv.2004.07.001

4. Centers for Disease Control and Prevention. Lyme and other tickborne diseases increasing. April 22, 2019 [cited 2020 Feb 1]. https://www.cdc.gov/ncezid/dvbd/media/lymetickborne-diseases-increasing.html

5. Joseph JT, Roy SS, Shams N, Visintainer P, Nadelman RB, Hosur S, et al. Babesiosis in lower Hudson Valley, New York, USA. Emerg Infect Dis. 2011;17:843-7. https:/ / doi.org/ 10.3201/eid1705.101334

6. Ostfeld RS, Levi T, Keesing F, Oggenfuss K, Canham CD. Tick-borne disease risk in a forest food web. Ecology. 2018;99:1562-73. https://doi.org/10.1002/ecy.2386

7. Hahn MB, Jarnevich CS, Monaghan AJ, Eisen RJ. Modeling the geographic distribution of Ixodes scapularis and Ixodes pacificus (Acari: Ixodidae) in the contiguous United States. J Med Entomol. 2016;53:1176-91. https://doi.org/10.1093/ jme/tjw076

8. Centers for Disease Control and Prevention). Babesiosis surveillance-18 states, 2011. MMWR Morb Mortal Wkly Rep. 2012;61:505-9.

9. Gray EB, Herwaldt BL. Babesiosis surveillance-United States, 2011-2015. MMWR Surveill Summ. 2019;68:1-11. https://doi.org/10.15585/mmwr.ss6806a1

10. Healthcare Cost and Utilization Project. Overview of the HCUP Nationwide Impatient Sample. 2011 [cited 2021 Jan 29]. https://www.hcup-us.ahrq.gov/db/nation/nis/ Overview_of_NIS_1998.pdf

11. HCUP National Inpatient Sample (NIS). Healthcare Cost and Utilization Project (HCUP). Overview of the HCUP Nationwide Impatient Sample. 2012. Agency for Healthcare Research and Quality. 2012 [cited 2021 Jan 29]. https://www.hcup-us.ahrq.gov/nisoverview.jsp

12. Healthcare Cost and Utilization Project. HCUP NIS Description of data elements. September 2008 [cited 2021 Jan 29]. https:/ / www.hcup-us.ahrq.gov/db/vars/ hosp_bedsize/nisnote.jsp

13. Burgess MJ, Rosenbaum ER, Pritt BS, Haselow DT, Ferren KM, Alzghoul BN, et al. Possible transfusiontransmitted Babesia divergens-like/MO-1 infection in an Arkansas patient. Clin Infect Dis. 2017;64:1622-5. https:/ / doi.org/10.1093/cid/cix216
14. Bloch EM, Herwaldt BL, Leiby DA, Shaieb A, Herron RM, Chervenak $\mathrm{M}$, et al. The third described case of transfusiontransmitted Babesia duncani. Transfusion. 2012;52:1517-22. https://doi.org/10.1111/j.1537-2995.2011.03467.x

15. Herwaldt BL, Kjemtrup AM, Conrad PA, Barnes RC, Wilson M, McCarthy MG, et al. Transfusion-transmitted babesiosis in Washington state: first reported case caused by a WA1-type parasite. J Infect Dis. 1997;175:1259-62. https://doi.org/10.1086/593812

16. Herc E, Pritt B, Huizenga T, Douce R, Hysell M, Newton D, et al. Probable locally acquired Babesia divergens-like infection in woman, Michigan, USA. Emerg Infect Dis. 2018;24:1558-60. https://doi.org/10.3201/eid2408.180309

17. White DJ, Talarico J, Chang H-G, Birkhead GS, Heimberger T, Morse DL. Human babesiosis in New York state: review of 139 hospitalized cases and analysis of prognostic factors. Arch Intern Med. 1998;158:2149-54. https://doi.org/10.1001/archinte.158.19.2149

18. Djokic V, Primus S, Akoolo L, Chakraborti M, Parveen N. Age-related differential stimulation of immune response by Babesia microti and Borrelia burgdorferi during acute phase of infection affects disease severity. Front Immunol. 2018;9:2891.

19. Shaw AC, Joshi S, Greenwood H, Panda A, Lord JM. Aging of the innate immune system. Curr Opin Immunol. 2010;22:507-13. https:/ / doi.org/10.1016/j.coi.2010.05.003

20. Centers for Disease Control and Prevention. Blacklegged tick (Ixodes scapularis) surveillance [cited 2021 Jun 29]. https:/ / www. cdc.gov/ticks/surveillance/BlackleggedTick.html

21. Menis M, Whitaker BI, Wernecke M, Jiao Y, Eder A, Kumar S, et al. Babesiosis occurrence among United States Medicare beneficiaries, ages 65 and older, during 2006-2017: overall and by state and county of residence. Open Forum Infect Dis. 2020;8:a608. https:// doi.org/10.1093/ofid/ofaa608

22. Krause PJ, Auwaerter PG, Bannuru RR, Branda JA, Falck-Ytter YT, Lantos PM, et al. Clinical practice guidelines by the Infectious Diseases Society of America (IDSA): 2020 guideline on diagnosis and management of babesiosis. Clin Infect Dis. 2021;72:e49-64. https:// doi.org/10.1093/cid/ ciaa1216

23. Spielman A. The emergence of Lyme disease and human babesiosis in a changing environment. Ann N Y Acad Sci. 1994;740(1 Disease in Ev):146-56. https:/ / doi.org/ 10.1111/j.1749-6632.1994.tb19865.x

24. Rodgers SE, Mather TN. Human Babesia microti incidence and Ixodes scapularis distribution, Rhode Island, 1998-2004. Emerg Infect Dis. 2007;13:633-5. https:/ / doi.org/10.3201/ eid1304.061035

25. Diuk-Wasser MA, Liu Y, Steeves TK, Folsom-O'Keefe C, Dardick KR, Lepore T, et al. Monitoring human babesiosis emergence through vector surveillance New England, USA. Emerg Infect Dis. 2014;20:225-31. https:/ / doi.org/10.3201/ eid2002.130644

26. Walter KS, Pepin KM, Webb CT, Gaff HD, Krause PJ, Pitzer VE, et al. Invasion of two tick-borne diseases across New England: harnessing human surveillance data to capture underlying ecological invasion processes. Proc Biol Sci. 2016;283:20160834.

27. Hatcher JC, Greenberg PD, Antique J, Jimenez-Lucho VE. Severe babesiosis in Long Island: review of 34 cases and their complications. Clin Infect Dis. 2001;32:1117-25. https://doi.org/10.1086/319742

28. Krause PJ, Gewurz BE, Hill D, Marty FM, Vannier E, Foppa IM, et al. Persistent and relapsing babesiosis in immunocompromised patients. Clin Infect Dis. 2008; 46:370-6. https:// doi.org/10.1086/525852 
29. Mareedu N, Schotthoefer AM, Tompkins J, Hall MC, Fritsche TR, Frost HM. Risk factors for severe infection, hospitalization, and prolonged antimicrobial therapy in patients with babesiosis. Am J Trop Med Hyg. 2017;97:121825. https://doi.org/10.4269/ajtmh.17-0146

30. Rosner F, Zarrabi MH, Benach JL, Habicht GS. Babesiosis in splenectomized adults. Review of 22 reported cases. Am J Med. 1984;76:696-701. https:/ / doi.org/10.1016/ 0002-9343(84)90298-5

31. Krause PJ, Telford SR III, Spielman A, Sikand V, Ryan R, Christianson D, et al. Concurrent Lyme disease and babesiosis. Evidence for increased severity and duration of illness. JAMA. 1996;275:1657-60. https://doi.org/10.1001/ jama.1996.03530450047031

32. Krause PJ, McKay K, Thompson CA, Sikand VK, Lentz R, Lepore T, et al.; Deer-associated Infection Study Group. Disease-specific diagnosis of coinfecting tickborne zoonoses: babesiosis, human granulocytic ehrlichiosis, and Lyme disease. Clin Infect Dis. 2002;34:1184-91. https://doi.org/10.1086/339813

33. Steere AC, McHugh G, Suarez C, Hoitt J, Damle N, Sikand VK. Prospective study of coinfection in patients with erythema migrans. Clin Infect Dis. 2003;36:1078-81. https://doi.org/10.1086/368187

34. Kugeler KJ, Griffith KS, Gould LH, Kochanek K, Delorey MJ, Biggerstaff BJ, et al. A review of death certificates listing Lyme disease as a cause of death in the United States. Clin Infect Dis. 2011;52:364-7. https:/ / doi.org/ $10.1093 /$ cid/ciq157

35. Agency for Healthcare Research and Quality. 1996 HCUP Nationwide Inpatient Sample (NIS) Design Report. 1996 [cited 2021 Jul 26]. https:/ / www.hcup-us.ahrq.gov/db/ nation/nis/reports/NIS_1996_Design_Report.jsp

36. Coffey R, Barrett M, Houchens R, Moy E, Ho K, Andrews R, et al. Methods applying AHRQ Quality Indicators to the Cost and Utilization Project (HCUP) data for the Seventh (2009) National Healthcare Disparities Report. Rockville (MD): US Agency for Healthcare Research and Quality; 2009.

Address for correspondence: Evan M. Bloch, Johns Hopkins University School of Medicine, Department of Pathology, 600 N Wolfe St/Carnegie 446D1, Baltimore, MD 21287, USA; email: ebloch2@jhmi.edu 